\title{
ON A COMMENT OF DOUGLAS CONCERNING WIDOM'S THEOREM
}

\author{
Mícheál Ó Searcóid
}

\section{$\S 1$ Introduction.}

Douglas, after presenting an adaptation of Widom's proof [2] that every Toeplitz operator has connected spectrum, comments, "Despite the elegance of the preceding proof of connectedness, we view it as not completely satisfactory for two reasons: First, the proof gives us no hint as to why the result is true. Second, the proof seems to depend on showing that the set of some kind of singularities for a function of two complex variables is connected ... " [1, p.196]. The purpose of this note is to demonstrate that one of the variables referred to by Douglas can be effectively suppressed by extensive use of the F. \& M. Riesz Theorem; the modified proof is, I believe, somewhat cleaner.

\section{$\S 2$ Preliminary concepts.}

The items in this section are well-known and are covered in [1].

Notation. The unit circle is denoted by $\mathbf{T}$. We consider the spaces $L^{p}=L^{p}(\mathbf{T})$ for $p=1,2, \infty$, where the measure is Lebesgue measure and the vectors are treated as functions defined almost everywhere. The functions $e_{n}: z \rightarrow z^{n}(n \in \mathbf{Z})$ form an orthonormal basis for the Hilbert space $L^{2}$. The Hardy spaces $H^{p}$ are $H^{p}=\left\{f \in L^{p}: \int_{\mathrm{T}} f e_{n}=0 \forall n>0\right\},(p=1,2, \infty) . P$ will denote the orthogonal projection from $L^{2}$ onto $H^{2}$. Note that $L^{\infty}$ and $H^{\infty}$ are Banach algebras, that $L^{\infty} \subset L^{2} \subset L^{1}$ and $H^{\infty} \subset H^{2} \subset H^{1}$ and that $L^{\infty} L^{2}=L^{2}$. For $\phi \in L^{\infty}, \sigma(\phi)$ will denote the spectrum, $\left\{\lambda \in \mathbf{C}: \phi-\lambda\right.$ not invertible in $\left.L^{\infty}\right\}$, of $\phi$ in $L^{\infty}$; note that this is the same as the essential range of $\phi$, namely, the set of all $\lambda \in \mathbf{C}$ 
such that, for every $\epsilon>0$, the set $\{z \in \mathbf{T}:|\phi(z)-\lambda|<\epsilon\}$ has positive measure. For $T \in \mathcal{B}\left(H^{2}\right)$, the algebra of bounded linear operators on $H^{2}, \sigma(T)$ will denote the spectrum of $T$ in $\mathcal{B}\left(H^{2}\right)$.

Definition. For each $\phi \in L^{\infty}$ we define the Toeplitz operator $T_{\phi} \in \mathcal{B}\left(H^{2}\right)$ by $T_{\phi} f=P(\phi f)$ for each $f \in H^{2}$.

Proposition 1. Suppose $f \in L^{1}$ and $\int_{\mathbf{T}} f e_{n}=0$ for all $n \in \mathbf{Z}$. Then $f=0$.

Proposition 2. Suppose $f, g \in H^{2}$. Then $f g \in H^{1}$.

Proposition 3. Suppose $\phi \in L^{\infty}$. Then $T_{\bar{\phi}}=T_{\phi}^{*}$.

Proposition 4. Suppose $\phi \in L^{\infty}$. Then $\sigma(\phi) \subseteq \sigma\left(T_{\phi}\right)$. (This implies, of course, that, if $T_{\phi}$ is invertible, then so is $\phi$. However, it is worth noting that the inverse of $T_{\phi}$ is not, except in very special cases, equal to $T_{\phi^{-1}}$.)

F. \& M. Riesz Theorem. Suppose $f \in H^{2}$. If $f \neq 0$ then the set of zeroes of $f$ has zero measure. (It follows immediately from this that if $\phi \in H^{\infty}$ and the essential range of $\phi$ is countable, then $\phi$ is essentially constant.)

\section{$\S 3$ The connectedness.}

Proposition 5. Suppose $\Gamma$ is a simple closed integration path and $K$ is a compact subset of the complex plane with $K \cap \Gamma=\emptyset$. Let $\phi \in L^{\infty}$ be such that $\sigma(\phi)=K$. Then $\Gamma$ fails to separate $K$ if and only if

$$
\exp \left(P \int_{\Gamma} \frac{d \mu}{\phi-\mu}\right)=e_{0}
$$

Proof: $\Gamma$ fails to separate $\sigma(\phi)$ if and only if the winding number function in $L^{\infty}$

$$
w(\Gamma, \phi)=\frac{1}{2 \pi i} \int_{\Gamma} \frac{d \mu}{\mu-\phi}
$$

is (essentially) constant. Since $w(\Gamma, \phi)$ has only integer values, the F. \& M. Riesz Theorem ensures that this happens if and only if $w(\Gamma, \phi)$ is in $H^{\infty}$, i.e., if and only if

$$
P \int_{\Gamma} \frac{d \mu}{\mu-\phi}=\int_{\Gamma} \frac{d \mu}{\mu-\phi}
$$


Since $\exp \left(\int_{\Gamma} \frac{d \mu}{\phi-\mu}\right)=e_{0}$, the result follows by invoking the F. \& M. Riesz Theorem again.

Proposition 6. Suppose $\phi \in L^{\infty}$ and $T_{\phi}$ is invertible in $\mathcal{B}\left(H^{2}\right)$. If $f \in H^{2}$ satisfies $T_{\phi} f=e_{0}$, then $f^{-1} \in H^{2}$ and $T_{\phi^{-1}} f^{-1}=e_{0}$.

Proof: Firstly, since $T_{\phi}$ is invertible, Propositions 3 and 4 ensure that $\phi$ and $\bar{\phi}$ are invertible in $L^{\infty}$ and that $T_{\bar{\phi}}=T_{\phi}^{*}$ is invertible in $\mathcal{B}\left(H^{2}\right)$. In particular, there is exactly one vector mapped to $e_{0}$ by $T_{\bar{\phi}}$, so that $\operatorname{dim}\left(\bar{\phi} H^{2} \cap \overline{H^{2}}\right)=1$. It follows that the space $H^{2} \cap \bar{\phi}^{-1} \overline{H^{2}}$ has dimension 1 and then also that

$$
\operatorname{dim}\left(\phi^{-1} H^{2} \cap \overline{H^{2}}\right)=1 .
$$

We deduce that $T_{\phi^{-1}}$ is injective and that there exists $g \in H^{2}$ such that $T_{\phi^{-1}} g=e_{0}$. Then there exist $u, v \in\left(H^{2}\right)^{\perp}$ such that $\phi f=e_{0}+u$ and $\phi^{-1} g=e_{0}+v$. By multiplication we have $f g=e_{0}+u+v+u v$, whence $u+v+u v \in H^{1}$ by Proposition 2 . Since $u, v \in\left(H^{2}\right)^{\perp}$, an easy calculation using Proposition 1 shows that $u+v+u v=0$ and hence that $f g=e_{0}$.

Widom's Theorem. Suppose $\phi \in L^{\infty}$; then $\sigma\left(T_{\phi}\right)$ is connected.

Proof: Consider the function $f: \mathbf{C} \backslash \sigma\left(T_{\phi}\right) \rightarrow H^{2}$ given by the equations $f(\lambda)=\left(\lambda-T_{\phi}\right)^{-1} e_{0}$. Then $f$ is differentiable and we have $P\left[(\lambda-\phi) f^{\prime}(\lambda)+f(\lambda)\right]=0$. But Proposition 6 gives also the equation $P[1 /((\lambda-\phi) f(\lambda))]=e_{0}$. Multiplying, we get the differential equation

$$
f^{\prime}(\lambda)=f(\lambda) P\left(\frac{1}{\phi-\lambda}\right) .
$$

Note that any non-zero solution of this equation is a multiple of $f$ by a non-zero function independent of $\lambda$. So, using the F. \& M. Riesz Theorem again, we solve to get, for any fixed $\alpha$ in each connected component of $\mathbf{C} \backslash \sigma\left(T_{\phi}\right)$ and for each $\lambda$ in that component,

$$
f(\lambda)=f(\alpha) \exp \left(P \int_{\Gamma} \frac{d \mu}{\phi-\mu}\right)
$$


where $\Gamma$ is any simple integration arc in the component going from $\alpha$ to $\lambda$. If $\Gamma$ is closed, the condition

$$
\exp \left(P \int_{\Gamma} \frac{d \mu}{\phi-\mu}\right)=e_{0}
$$

of Proposition 5 holds, so no such $\Gamma$ separates $\sigma(\phi)$ and connectedness of $\sigma\left(T_{\phi}\right)$ will follow if we can show that $\sigma\left(T_{\phi}\right)$ is exterior to such a $\Gamma$ whenever $\sigma(\phi)$ is. Suppose, then, that $\Gamma$ is a simple closed integration path in $\mathbf{C} \backslash \sigma\left(T_{\phi}\right)$ and that $\sigma(\phi)$ is exterior to $\Gamma$. Then the solution to the differential equation gives a unique analytic continuation of $f$ to the interior of $\Gamma$, so that, setting $Q$ to be the associated spectral idempotent for $T_{\phi}$, we have

$$
Q e_{0}=\frac{1}{2 \pi i} \int_{\Gamma}\left(\mu-T_{\phi}\right)^{-1} e_{0} d \mu=\frac{1}{2 \pi i} \int_{\Gamma} f(\mu) d \mu=0
$$

Now $\left(\lambda-T_{\phi}\right)\left(e_{n} f(\lambda)\right)=e_{n}+\sum_{i=0}^{n-1} \beta_{i} e_{i}$ for each $\lambda \in \Gamma$ and some related scalars $\beta_{i}$; assuming inductively that $Q e_{i}=0$ for $i<n$, it follows, since $Q$ commutes with $T_{\phi}$, that

$$
Q\left(e_{n} f(\lambda)\right)=\left(\lambda-T_{\phi}\right)^{-1} Q e_{n}
$$

and integration around $\Gamma$ gives $Q e_{n}=0$. So $Q=0$ by induction, whence no part of $\sigma\left(T_{\phi}\right)$ is interior to $\Gamma$ and the theorem is proved.

\section{References}

[1] Douglas, R. G., Banach Algebra techniques in Operator Theory, Academic Press, New York \& London, 1972.

[2] Widom, H., On the spectrum of a Toeplitz operator, Pacific. J. Math., 14 (1964), 365-375.

Mícheál Ó Searcóid

Department of Mathematics

University College

Dublin

email: micheal.osearcoid@ucd.ie 